\title{
BMJ Open The association between the use of biomedical services and the holistic use of traditional East Asian medicine: a national survey of outpatients in South Korea
}

Jae-Mahn Shim, ${ }^{1}$ Yun-Suk Lee ${ }^{2}$

To cite: Shim J-M, Lee Y-S. The association between the use of biomedical services and the holistic use of traditional East Asian medicine: a national survey of outpatients in South Korea. BMJ Open 2017;7:e018414. doi:10.1136/ bmjopen-2017-018414

- Prepublication history and additional material for this paper are available online. To view these files, please visit the journal online (http://dx.doi. org/10.1136/bmjopen-2017018414).

Received 28 June 2017 Revised 30 October 2017 Accepted 10 November 2017

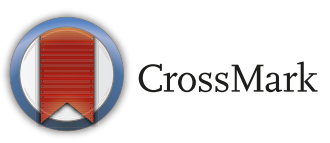

${ }^{1}$ Department of Sociology, Korea University, Seoul, Republic of Korea

${ }^{2}$ Department of Sociology, University of Seoul, Seoul, Republic of Korea

Correspondence to Professor Jae-Mahn Shim; jaemahn.shim@gmail.com

\section{ABSTRACT}

Objectives The holistic use of a system of complementary and alternative medicine (CAM) is potentially linked to its treatment outcomes. This paper examines how the use of biomedicine is associated with the holistic use of CAM, focusing on traditional East Asian medicine (EM) that is uniquely integrated in the medical system in South Korea. Design/Settings A representative national sample of EM outpatients in South Korea.

Participants 3861 survey respondents.

Methods By using the 2011 Korean National Survey of EM patients, ordered logistic regression models specify the relationship between EM outpatients' use of biomedicine and their holistic use of EM modalities.

Results Among EM outpatients who used at least one EM modality in the past 3 months, people who used two $(33.3 \%)$ or three $(29.4 \%)$ modalities together are the two highest proportions, followed by users of four $(18.1 \%)$, five (7.2\%), six (2.1\%) and seven (0.6\%) modalities. The odds for EM users to use EM holistically are $17 \%$ greater among EM users who used biomedicine as well, compared with EM users who did not use biomedicine.

Conclusions The healthcare community should recognise that CAM use likely becomes holistic as people use biomedicine concomitantly, when the practice rights over a CAM system are comprehensively and exclusively entitled to a group of CAM professionals who are independent from practitioners of biomedicine.

\section{INTRODUCTION}

The presence of complementary and alternative medicine (CAM) is substantial in contemporary healthcare systems around the world. ${ }^{12}$ The healthcare community is concerned with the ways in which CAM is integrated into mainstream biomedicine ${ }^{34}$ and subsequent healthcare outcomes that CAM produces. Studies suggest that CAM services be provided holistically so that CAM users can utilise all the related treatment modalities within a whole system of CAM. ${ }^{56}$ In the literature, the holistic use of a CAM system refers
Strengths and limitations of this study

- The study comprises a national sample of East Asian medicine (EM) users who visited EM facilities across South Korea.

- It specifies several multivariate ordered logistic regression models to support the findings.

- All the measures are based on self-reports of survey participants.

to the utilisation behaviour of CAM users who use two or more treatment modalities together that constitute the CAM system. ${ }^{78}$ In selective and fragmented use, on the contrary, people use only a certain modality of the CAM system and not the other modalities. It is argued that the holistic use can maximise the treatment effects of CAM compared with the selective and fragmented use of only one modality out of multiple interrelated modalities in the whole system. ${ }^{8}$

This paper aims to investigate what generates this difference in the behaviour of CAM users. Drawing on the literature of medical systems, the paper posits that the ways in which CAM is institutionalised in medical systems are consequential for the extent to which CAM users utilise various treatment modalities of a CAM system holistically. In accordance with previous studies, ${ }^{5-8}$ the paper defines the holistic use of CAM as CAM users' utilisation behaviour in which two or more treatment modalities of a CAM system are used together rather than a single modality being used in isolation from the other modalities of the CAM system. When a user resorts to more modalities, their behaviour is interpreted to be more holistic.

The paper pursues this investigation by examining the case of a whole system of traditional East Asian medicine (EM) that 
is composed of multiple treatment modalities, such as acupuncture, moxibustion, cupping, herbal remedies and acupressure. In particular, the paper develops a specific hypothesis that elaborates the unique institutional condition of EM in South Korea and relates it to the utilisation behaviour of EM users who may also use biomedical services concomitantly that are readily available in the national healthcare system of the country. The hypothesis focuses on how the use of biomedical services is associated with the holistic use of EM in the Korean context.

In Korea, the professional practice of EM is comprehensively and exclusively performed by EM doctors and is separate from the professional practice of biomedicine performed by medical doctors. ${ }^{9-12}$ Korea features distinctive systems of education and licensure for EM doctors who are legally permitted to practice the whole range of modalities of EM, independent from medical doctors of biomedicine. The EM doctors even hold the right to practice all the EM modalities in such an exclusive way that doctors of biomedicine do not hold the right to practice any of the EM modalities. Reciprocally, these EM doctors are not allowed to practice biomedicine. This comprehensive distinction of all treatment modalities of EM within the medical system and, at the same time, the exclusive entitlement of the practice rights of these modalities only to EM doctors (and not biomedical doctors), likely portrays EM to medical service users as a system of medical practices that is distinct and independent from biomedicine. EM is also viewed as a whole medical system that is composed of a variety of interrelated treatment modalities that are readily available for the needs of medical service users.

In this institutional condition in Korea, EM users who also use biomedical services are likely those who search for diverse medical resources of different kinds that the national medical system provides for them. These EM users, when compared with EM users who do not use biomedical services and thus do not seek diverse medical resources, are likely to look for even more diverse modalities that are available within EM itself. Therefore, this paper hypothesises that the EM users who also use biomedical services are likely to use EM more holistically.

Hypothesis: Among EM users in Korea where certified EM professionals hold the comprehensive and exclusive practice rights over EM, medical service users' utilisation of biomedical services is positively associated with their likelihood of using EM holistically.

\section{METHODS}

Data come from the 2011 Korean National Survey of EM Patients (NSEMP) that was administered to a nationally representative sample of patients who visited (ie, outpatients) or were hospitalised (ie, inpatients) in an EM facility as of September 2011. This survey used the national sampling frame of $12250 \mathrm{EM}$ facilities that were registered in the national health insurance system. This sampling frame was duly regarded as the national population of EM facilities in Korea, since all medical service providers should be registered with the national insurance system for reimbursement from the national government in the universal Korean healthcare system. The survey then drew a stratified systematic sample of $471 \mathrm{EM}$ facilities (4\% of the sampling frame). At each selected facility, the outpatient questionnaire of the survey was administered to a random sample of nine outpatients drawn from people in the waiting area. When the facility was equipped with hospital beds, an additional random sample of eight inpatients was drawn from the list of inpatients and these inpatients participated in the inpatient questionnaire. As a result, 3926 outpatients and 1581 inpatients participated in the survey.

This paper analyses only the responses from the outpatient EM users, since only the outpatient questionnaire investigated the uses of EM in each of the seven different EM modalities in detail; the inpatient questionnaire investigated whether a respondent ever used any of the EM modalities without differentiating which modality was used. These inpatient responses give no information on which and how many EM modalities were used and, thus, how holistic the EM use was. As a result, the following analysis includes responses from the final sample of 3861 outpatient EM users. The difference from the total number of outpatient participants $(65=3926-3861)$ is due to a further exclusion of 65 inpatient respondents for whom there were missing values for one or more of the variables that are included in the following analysis. This study, which uses publically available survey data, is granted an exemption from requiring ethics approval by the Institutional Review Board of Korea University.

The dependent variable, the holistic use of $E M$, is an ordinal variable that measures the number of different EM modalities that were used together by a respondent in the past 3 months. It is a composite measure that summarises responses to seven distinct questions. Each of the seven questions asked whether a respondent used one of the seven EM modalities respectively ('have you used (a specific EM modality) for medical problems in the past 3 months?'), such as (1) herbal extracts, (2) herbal pills/ powders, (3) acupuncture, (4) moxibustion, (5) cupping, (6) chuna and (7) manual treatments. The response to each question is coded 1 if yes ( 0 if not). Thus, the values of the dependent variable range from 1 to 7 . The focal independent variable is the use of biomedical services, which measures whether a respondent visited a biomedical clinic or hospital where biomedical doctors provide medical services for the medical conditions for which the respondent used EM modalities. It is coded 1 if a respondent visited a biomedical clinic or hospital (0 if not).

A set of potential covariates, which may intervene in the relationship between holistic EM use and the use of biomedical services, are incorporated in the analysis as control variables. These control variables are the frequency of EM use, self-rated health status, gender, age, marital status, the highest level of education, and monthly 


\begin{tabular}{|c|c|c|c|c|c|}
\hline Variable & $\mathbf{N}$ & $\%$ & Variable & $\mathbf{N}$ & $\%$ \\
\hline The holistic use of EM & & & Age & & \\
\hline 2 & 1284 & 33.3 & 20 s or 30 s & 894 & 23.2 \\
\hline 3 & 1135 & 29.4 & $40 s$ & 762 & 19.7 \\
\hline 5 & 276 & 7.2 & $60 s$ & 575 & 14.9 \\
\hline 6 & 82 & 2.1 & $70 \mathrm{~s}$ & 522 & 13.5 \\
\hline 7 & 22 & 0.6 & 80 s or more & 132 & 3.4 \\
\hline The use of biomedical services & & & Marital status & & \\
\hline Yes & 1641 & 42.5 & Single & 613 & 15.9 \\
\hline 4 to 10 times & 1289 & 33.4 & Education & & \\
\hline 11 to 30 times & 885 & 22.9 & No formal education & 279 & 7.2 \\
\hline 30 times or more & 298 & 7.7 & Elementary school & 557 & 14.4 \\
\hline Self-rated health status & & & Middle school & 467 & 12.1 \\
\hline Very bad & 103 & 2.7 & High school & 1215 & 31.5 \\
\hline Bad & 1010 & 26.2 & College or graduate school & 1343 & 34.8 \\
\hline Fair & 1651 & 42.8 & Household income & & \\
\hline Good & 983 & 25.5 & less than 1 million wons & 733 & 19.0 \\
\hline
\end{tabular}

${ }^{*}$ The socio-demographic characteristics of EM users show that EM is being used very widely across different social groups in age, education and income.

†This refers to how many times a respondent has used EM services in the past 3 months. It is certain that, when a respondent uses EM less often, the respondent is less likely to use different treatment modalities of EM. However, a respondent who uses EM very often does not necessarily utilise different treatment modalities (eg, a respondent who uses only acupuncture many times).

EM, East Asian medicine.

household income (table 1 for descriptive statistics). This paper uses ordered logistic regression models to test the hypothesis about the relationship between holistic EM use and the use of biomedical services, since it interprets different values for the dependent variable as ordered categories that refer to the extent of holistic EM use. Results from negative binomial regression models, which treat the dependent variable as a count measure, agree with the results reported here. For comparison, results from negative binomial regression models are reported in Table A1 in the online supplementary appendix 1.

\section{RESULTS}

Among all current outpatient EM users who used at least one EM modality in the past 3 months, EM users who used two or three modalities are the two greatest in number, followed by those who used four, five, six, and seven modalities. The proportion of EM users who used only one modality is only $9.4 \%$. It turns out that more than $90 \%$ of current EM outpatients in Korea used multiple EM modalities together when they ever resorted to EM.

Close to a half of these EM users $(42.5 \%)$ also used biomedical services by visiting a biomedical clinic or hospital. In addition, the bivariate tabulation on the relationship between the extent of holistic EM use and the use of biomedical services shows that there is a positive relationship between the two; EM users who utilised various EM modalities more holistically were more likely to use biomedical services as well (table 2). Put differently, EM users who also used biomedical services were more likely to utilise EM modalities holistically than EM users who did not use biomedical services. 
Table 2 The bivariate association between the use of biomedical services and the holistic use of traditional East Asian Medicine (EM)

\begin{tabular}{llrrr} 
& \multicolumn{4}{l}{ The use of biomedical services } \\
\cline { 2 - 5 } & \multicolumn{1}{l}{ No } & \multicolumn{1}{l}{ Yes } & \multicolumn{1}{c}{ Total } \\
\hline The holistic & 1 & $227(62.5)$ & $136(37.5)$ & $363(100.0)$ \\
use of EM & 2 & $766(59.7)$ & $518(40.3)$ & $1284(100.0)$ \\
(No. of EM & 3 & $654(57.6)$ & $481(42.4)$ & $1135(100.0)$ \\
modalities & 3 & $389(55.6)$ & $310(44.4)$ & $699(100.0)$ \\
used) & 4 & $138(50.0)$ & $138(50.0)$ & $276(100.0)$ \\
& 5 & $39(47.6)$ & $43(52.4)$ & $82(100.0)$ \\
& 6 & $7(31.8)$ & $15(68.2)$ & $22(100.0)$ \\
& 7 & $2220(57.5)$ & $1641(42.5)$ & $3861(100.0)$ \\
\hline
\end{tabular}

Note: percentages in parentheses. Pearson's $\chi^{2}(6)=22.8001$; $P$ value, 0.001 .

Model one in table 3 puts this bivariate association in ORs and finds it to be statistically significant. The odds for EM users who also used biomedical services to utilise EM holistically are greater than those for EM users who did not use biomedical services $(\mathrm{OR}=1.27 ; 95 \% \mathrm{CI}=1.13$ to 1.42$)$. The two subsequent models show that this association remains the same when respondents' frequency of EM use is controlled (model 2) or when respondents' health status is controlled (model 3). The final model 4 incorporates these two confounders together and other possible confounders as control variables; the positive association between holistic EM use and the use of biomedical services still persists. In this final model, the odds for EM users who also used biomedicine to use EM holistically are $17 \%$ greater than those for EM users who did not use biomedicine $(\mathrm{OR}=1.17$; $95 \% \mathrm{CI}=1.04$ to 1.31$)$. These results support the hypothesis unambiguously.

\section{DISCUSSION}

This paper found that people who used biomedicine were more likely to use EM holistically in South Korea. Among EM outpatients who used at least one EM modality in the past 3 months, people who used two or three modalities together are the two greatest in number, followed by users of four, five, six and seven modalities. The odds for EM users to use EM holistically are greater among EM users who used biomedicine as well, compared with EM users who did not use biomedicine. It is a limitation of this study that it used measures based on self-reports of survey participants. However, it is worth noting that the paper used a national sample of EM users who visited EM facilities across South Korea and specified multivariate regression models to show the robustness of these findings.

To our knowledge, this is the first study in the global medical community that examines how the use of biomedical services is associated with the extent to which the users of CAM utilise various modalities of a CAM system holistically. It is surprising that this line of inquiry has been neglected in the literature, in spite of a potential link between the holistic versus selective utilisation of a CAM system and its healthcare outcomes. Furthermore, it is very probable that CAM users shape their specific ways of using CAM in relation to biomedical services that are available to them. In this sense, this paper contributes to developing a much-needed analytical perspective that elaborates CAM utilisation behaviour beyond existing studies of the simple utilisation rate and the popularity of CAM. ${ }^{13-19}$

This perspective has grown out of a group of studies that investigated the complementary versus substitutive relationship between CAM use and biomedicine use. ${ }^{920-23}$ Whereas these existing studies have investigated whether the utilisation of fragmented CAM modalities increases or decreases the utilisation of biomedical services by comparing the behaviour of CAM users to the behaviour of non-users, this paper originally focuses on CAM users only and investigates how their CAM utilisation behaviour is shaped by their use (vs. non-use) of biomedical services. In this sense, this paper provides CAM-centred evidence to the complementarity versus substitution debate; its finding suggests that CAM use can be complementary to biomedicine to the extent that users rely on a variety of treatment modalities within a CAM system even when they use biomedical services (ie, the holistic co-utilisation of CAM).

The findings in this paper suggest that the knowledge of how CAM is institutionalised in a medical system can generate reasonable predictions about how CAM users behave. It is known that the various modalities of EM are disconnected from one another in the Japanese medical system so that only herbal remedies are selectively incorporated into the practices of biomedical doctors ${ }^{24-28}$; acupuncture and acupressure are each relegated as pseudo-medicine to medical technicians, such as acupuncturists and massage therapists who are permitted to practice only acupuncture and massage therapies. These technicians are not allowed to practice herbal remedies or other EM modalities. Similar observations are made that the otherwise rich practices of acupuncture are truncated and simplified in the dominant biomedical healthcare systems in the $\mathrm{US}^{29-31}$ and the UK. ${ }^{32}$ In the contemporary French biomedical system, a variety of CAM systems are reported to become 'balkanized' and their constituent treatment modalities are torn apart from one another in practice. ${ }^{33}$ In these institutional contexts, this paper suggests the intersection of a CAM system with biomedicine can result in the fragmented and partial use of the CAM system as people use biomedical services concomitantly.

\section{CONCLUSIONS}

The healthcare community should recognise that CAM use likely becomes holistic as people use biomedicine concomitantly, when the practice rights over a CAM system are comprehensively and exclusively entitled to a group of CAM professionals who are independent from 
Table 3 Ordered logistic regression models of holistic East Asian medicine (EM) use on the use of biomedical services and other covariates

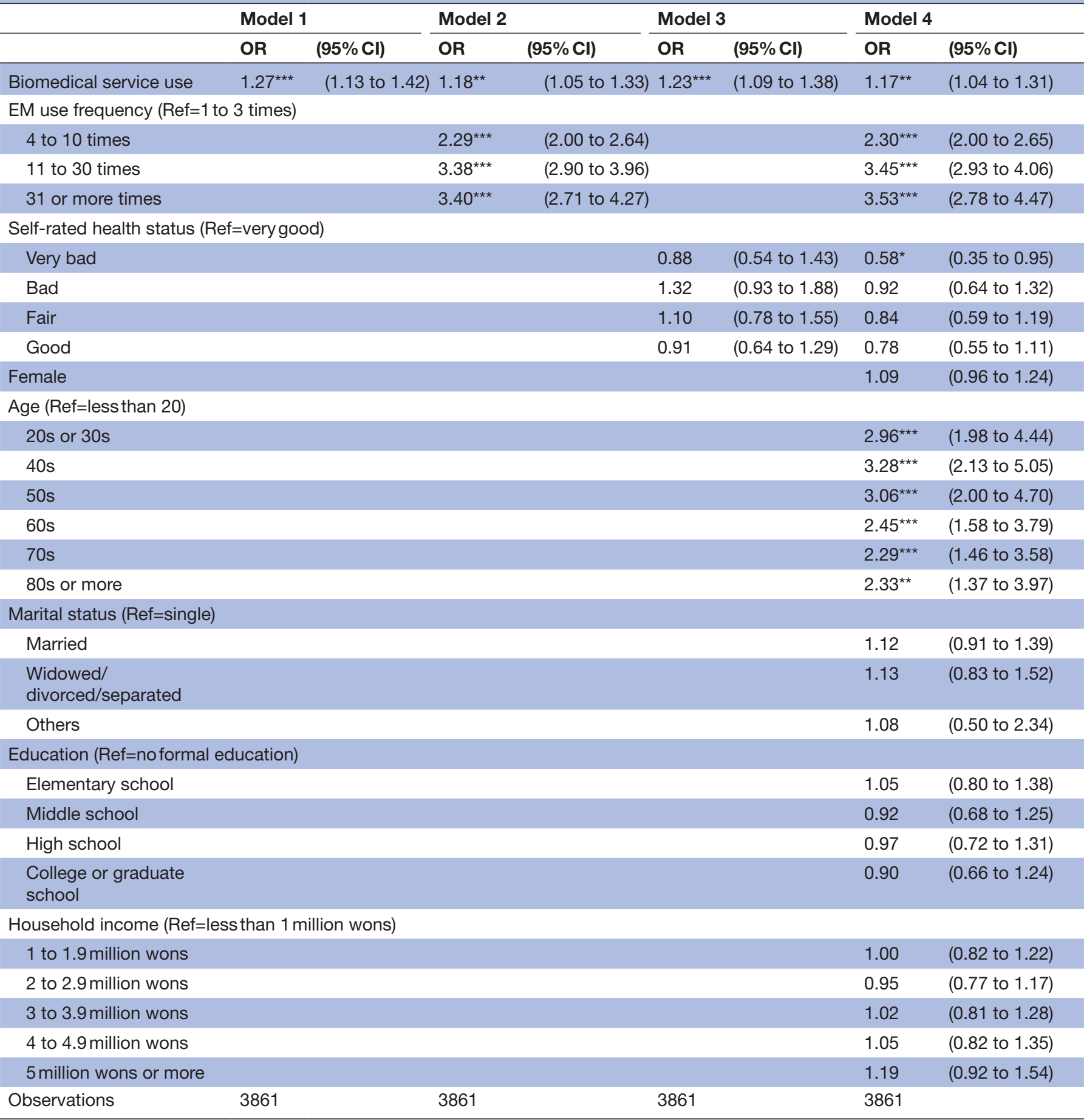

Note, significant at ${ }^{\star} 0.01,{ }^{\star \star} 0.01,{ }^{\star \star *} 0.001$.

practitioners of biomedicine. This conclusion is based on the experiences of EM users in Korea. It will take more research to generalise this finding against other institutional contexts and, at the same time, to discern any cross-national differences. A comparative study between the East Asian countries, such as China, Korea and Japan where EM originated and is institutionalised differently, will shed more light.
The authors recommend that the CAM-centred perspective, asking how CAM use is reconstructed by biomedicine use, is especially relevant to studying CAM utilisation behaviour in societies where a relatively coherent system of CAM has existed for quite some time (eg, traditional Indian medicine in the US, traditional African medicine in Africa, Ayurveda and Indian medical traditions in India, etc.). The international medical community will 
gain a lot from these future studies. It will gain even more from the studies when the link between the holistic versus selective use of CAM and its varying healthcare outcomes is examined further.

Acknowledgements This work was supported by the Ministry of Education of the Republic of Korea and the National Research Foundation of Korea (NRF-2015S1A5A8017517). The views in this paper are the authors' and should not be interpreted as those of the supporter. The authors thank Keith Woogerd for copyediting the manuscript.

Contributors JMS conceived the study, analysed the data, and wrote the manuscript. YSL helped conceive the study, modify the data analysis, and revise the manuscript.

Funding The first and corresponding author received financial support from the Ministry of Education of the Republic of Korea and the National Research Foundation of Korea (NRF-2015S1A5A8017517) for data analysis and manuscript writing.

Competing interests None declared.

Provenance and peer review Not commissioned; externally peer reviewed.

Data sharing statement The data are available from the corresponding author upon request.

Open Access This is an Open Access article distributed in accordance with the Creative Commons Attribution Non Commercial (CC BY-NC 4.0) license, which permits others to distribute, remix, adapt, build upon this work non-commercially, and license their derivative works on different terms, provided the original work is properly cited and the use is non-commercial. See: http://creativecommons.org/ licenses/by-nc/4.0/

(C) Article author(s) (or their employer(s) unless otherwise stated in the text of the article) 2017. All rights reserved. No commercial use is permitted unless otherwise expressly granted.

\section{REFERENCES}

1. World Health Organization. WHO traditional medicine strategy 20142023. Geneva, Switzerland: World Health Organization, 2013.

2. Bodeker G, Ong C-K, Grundy C, et al. WHO global atlas of traditional, complementary and alternative medicine. Kobe, Japan: WHO Centre for Health Development, 2005.

3. Xu J, Yang Y. Traditional Chinese' medicine in the Chinese health care system. Health Policy 2009;90:133-9.

4. Kaboru BB, Falkenberg T, Ndulo J, et al. Communities views on prerequisites for collaboration between modern and traditional health sectors in relation to STI/HIV/AIDS care in Zambia. Health Policy 2006;78:330-9.

5. MacPherson H, Nahin R, Paterson C, et al. Developments in acupuncture research: big-picture perspectives from the leading edge. J Altern Complement Med 2008;14:883-7.

6. Ritenbaugh C, Verhoef M, Fleishman S, et al. Whole systems research: a discipline for studying complementary and alternative medicine. Altern Ther Health Med 2003;9:32-6.

7. Shim JM, Kim J. Cross-national differences in the holistic use of traditional East Asian medicine in East Asia. Health Promot Int 2016:daw089.

8. Cao $\mathrm{Y}, \mathrm{Zhan} \mathrm{H}$, Pang J, et al. Individually integrated traditional Chinese medicine approach in the management of knee osteoarthritis: study protocol for a randomized controlled trial. Trials 2011;12:160.

9. Shim JM. The relationship between the use of complementary and alternative medicine and the use of biomedical services: evidence from East Asian medical systems. Asia Pac J Public Health 2016;28:51-60.

10. Holliday I. Traditional medicines in modern societies: an exploration of integrationist options through East Asian experience. $J$ Med Philos 2003;28:373-89.

11. Yoon K-J, Kim D-S. Ui-Hanui Kongsangbalchunul Wihan Tonghapchok Chopkeunbangsik Mosaek (Ways of Integrating Oriental Medicine and Western Medicine): Korea Institute of Health and Social Affairs, 2013 Seoul, Korea.

12. Dongwon S. How four different political systems have shaped the modernization of traditional Korean medicine between 1900 and 1960. Hist Sci 2008;17:225-41.

13. Frass M, Strassl RP, Friehs $\mathrm{H}$, et al. Use and acceptance of complementary and alternative medicine among the general population and medical personnel: a systematic review. Ochsner $J$ 2012;12:45-56.

14. Harris PE, Cooper KL, Relton C, et al. Prevalence of complementary and alternative medicine (CAM) use by the general population: a systematic review and update. Int J Clin Pract 2012;66:924-39.

15. Wong TW, Wong SL, Donnan SP. Prevalence and determinants of the use of traditional Chinese medicine in Hong Kong. Asia Pac J Public Health 1995;8:167-70.

16. Chen FP, Chen TJ, Kung YY, et al. Use frequency of traditional Chinese medicine in Taiwan. BMC Health Serv Res 2007;7:26.

17. Hori S, Mihaylov I, Vasconcelos JC, et al. Patterns of complementary and alternative medicine use amongst outpatients in Tokyo, Japan. BMC Complement Altern Med 2008;8:14.

18. Hong CD. Complementary and alternative medicine in Korea: current status and future prospects. J Altern Complement Med 2001;7(Suppl 1):33-40.

19. Jin L. From mainstream to marginal? Trends in the use of Chinese medicine in China from 1991 to 2004. Soc Sci Med 2010;71:1063-7.

20. Druss BG, Rosenheck RA. Association between use of unconventional therapies and conventional medical services. JAMA 1999;282:651-6.

21. van Gameren E. Health insurance and use of alternative medicine in Mexico. Health Policy 2010;98:50-7.

22. Ayers SL, Kronenfeld JJ. Delays in seeking conventional medical care and complementary and alternative medicine utilization. Health Serv Res 2012;47:2081-96.

23. Pagan JA, Pauly MV. Access to conventional medical care and the use of complementary and alternative medicine. Health Aff 2005;24:255-62.

24. Lock M. East Asian Medicine in Urban Japan: varieties of medical experience. Vol. 4. Berkeley: University of California Press, 1980.

25. Lock M. The organization and practice of East Asian medicine in Japan: continuity and change. Soc Sci Med Med Anthropol 1980;14B:245-53.

26. Katayama K, Yoshino T, Munakata K, et al. Prescription of kampo drugs in the Japanese health care insurance program. Evid Based Complement Alternat Med 2013;2013:1-7.

27. Kobayashi A, Uefuji M, Yasumo W. History and progress of Japanese acupuncture. Evid Based Complement Alternat Med 2010;7:359-65.

28. Tsutani K. The evaluation of herbal medicines: an East Asian perspective. In: Lewith GT, Aldridge D, eds. Clinical research methodology for complementary therapies. London: Hodder \& Stoughton, 1993:365-93.

29. Barnes LL. The acupuncture wars: the professionalizing of American acupuncture. Med Anthropol 2003;22:261-301.

30. Wolpe PR. The maintenance of professional authority: acupuncture and the American physician. Soc Probl 1985;32:409-24.

31. Braverman C, Baker C, Harris R. Acupuncture and Oriental Medicine (AOM) in the United States. Am Acupuncturist 2009;47:22-6.

32. Givati A, Hatton K. Traditional acupuncturists and higher education in Britain: the dual, paradoxical impact of biomedical alignment on the holistic view. Soc Sci Med 2015;131:173-80.

33. Ramsey M. Alternative medicine in modern France. Med Hist 1999;43:286-322. 